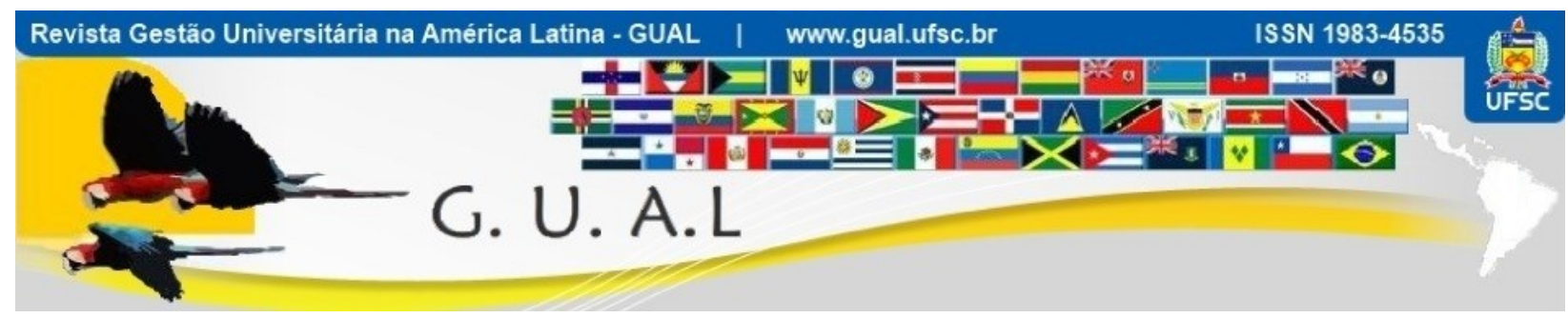

DOI: http://dx.doi.org/10.5007/1983-4535.2018v11n1p92

\title{
INFLUÊNCIA E DESEMPENHO DAS VARIÁVEIS ESPACIAIS EM INTERAÇÃO COM OS ASPECTOS PEDAGÓGICOS E ESTRUTURAIS NA SATISFAÇÃO DOS ALUNOS
}

\author{
INFLUENCE AND PERFORMANCE MEASUREMENTS OF SPACE IN \\ INTERACTION WITH EDUCATIONAL AND STRUCTURAL ISSUES IN \\ SATISFACTION OF PUPILS
}

Paulo Roberto da Cunha, Doutor Fundação Universidade Regional de Blumenau - FURB pauloccsa@gmail.com

Jaime Dagostim Picolo, Mestre Fundação Universidade Regional de Blumenau - FURB jaimepicolo@hotmail.com

Josiane Brighenti, Mestre Fundação Universidade Regional de Blumenau - FURB josianebrighenti@gmail.com

Recebido em 14/março/2016

Aprovado em 03/novembro/2017

Sistema de Avaliação: Double Blind Review

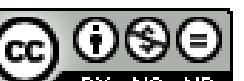

Esta obra está sob uma Licença Creative Commons Atribuição-Uso. 


\title{
RESUMO
}

O objetivo deste estudo consiste em verificar se os fatores das variáveis espaciais, em interação com os aspectos pedagógicos e estruturais, influenciam no nível de satisfação dos alunos e, na intenção de continuar estudando em uma Instituição de Ensino Superior. A população compreendeu os acadêmicos de graduação das fases finais do curso de administração com habilitação em comércio exterior de uma Instituição de Ensino Superior de Santa Catarina. A amostra é representada por 61 alunos que concordaram em participar da pesquisa, tomando tempo suficiente para responder as questões formuladas. Quanto a metodologia, a pesquisa caracteriza-se como descritiva, quantitativa, por meio de levantamento. Os resultados apontaram que os aspectos pedagógicos são os mais elementares e que mais influenciam na satisfação dos alunos da instituição estudada. Os aspectos estruturais e espaciais, de maneira geral, demonstraram ter relação com a satisfação, no entanto, não foram consideradas como importantes pelos alunos.

Palavras-chave: Variáveis Espaciais. Instituição de Ensino Superior. Satisfação.

\begin{abstract}
The aim of this study is to verify whether the factors of spatial variables in interaction with the pedagogical and structural, influence the students' level of satisfaction and the intention to continue studying at a higher education institution. The population comprised undergraduate students of the final stages of the course of administration with specialization in foreign trade of a Higher Education Institution of Santa Catarina. The sample is represented by 61 students who agreed to participate, taking enough time to answer the questions. As for methodology, the research is characterized as descriptive, quantitative, through survey. The results showed that the pedagogical aspects are the most basic and most influence on the satisfaction of the students of the study institution. The structural and spatial aspects, in general, have demonstrated relationship with satisfaction, however, were not considered important by the students.
\end{abstract}

Keywords: Spatial Variables. Institution of Higher Education. Satisfaction. 


\section{INTRODUÇÃO}

O interesse da sociedade pela educação superior tem aumentado significativamente nos últimos anos e, como reflexo, movimentou o mercado de oferta das instituições de ensino, ocasionando a expansão do ensino superior (COLOSSI, 2007). A expansão e variedade das Instituições de Ensino Superior - IES, sejam elas universidades, centros universitários, institutos ou faculdades integradas ou isoladas, bem como as modalidades de ensino presencial, semipresencial e à distância, impulsionaram o mercado de educação tornando-o mais competitivo, fazendo com que essas instituições atentassem às práticas de gestão, com isso, desenvolvessem estratégias e direcionassem esforços para atrair e conquistar "clientes" (MIRANDA, 2006).

Existem critérios que norteiam as práticas de gestão em instituições de ensino, são elas a eficiência considerada critério econômico, eficácia quanto ao critério pedagógico, efetividade em se tratando de critério político, e relevância como critério antropológico. A eficiência e eficácia são critérios de natureza instrumental e por isso devem subordinar-se aos critérios efetividade e relevância, enquanto critérios de natureza substantiva mais adequados às instituições de ensino (SANDER, 1984).

As organizações variam significativamente em muitos aspectos, tais como: tipos de clientes, tecnologias, habilidades dos trabalhadores, estruturas e estilos de coordenação e relacionamento com seu ambiente externo. Existem muitos elementos comuns na operação de faculdades e universidades, empresas, organizações governamentais, porém, nunca duas organizações são iguais. Além disso, as universidades são organizações singulares, e diferem na maioria dos seus aspectos das empresas industriais, empresas de serviços e organizações governamentais (BALDRIDGE et al.,1971).

No âmbito da gestão das instituições de ensino superior, esta pesquisa busca associar as variáveis espaciais e sua interação com aspectos pedagógicos e estruturais, para verificar a satisfação dos alunos e o que faz com que permaneçam na instituição escolhida. Segundo Bateson e Hoffman (2001), existe uma relação entre o nível de satisfação e a intenção de recompra dos clientes. Entretanto essa relação nem sempre é linear. Em ambientes com baixa competição como em monopólios, baixos níveis de satisfação são suficientes para reter clientes, pois o mesmo não tem alternativa de escolha como, por exemplo, hospitais, fornecedores de energia elétrica. Contudo, quanto maior for o grau de competição, maior a 
necessidade de investir em satisfação dos clientes, pois quanto maior o grau dessa satisfação, maior sua intenção de comprar novamente.

Turley e Milliman (2000), organizam os fatores de influência na decisão de compra no varejo com base nos estímulos ambientais ou atmosféricos, que podem afastar ou aproximar clientes, sendo que apresentou os seguintes grupos de fatores: Variáveis externas: são os fatores relacionados à parte externa da loja, como por exemplo, a fachada, a entrada da loja, as vitrines, o estacionamento e a arquitetura. Variáveis internas: são características do varejo relacionadas à pavimentação, iluminação, odores, sons, temperatura e a limpeza; Variáveis de layout e design: características relacionadas ao uso do espaço, largura dos corredores e a localização dos diversos departamentos; Variáveis de ponto de venda e decoração: Esta categoria inclui exposição de produtos, displays de ponto de venda, cartazes, placas, cartões, mensagens de tele texto e decorações de parede. Variáveis humanas: Esta categoria inclui a densidade de pessoas, a privacidade pessoal, as características dos clientes e as características dos funcionários.

Neste contexto, busca-se associar as variáveis espaciais com instituições de ensino superior, surge então a seguinte pergunta norteadora da pesquisa: os fatores das variáveis espaciais, em interação com os aspectos pedagógicos e estruturais, influenciam no nível de satisfação dos alunos e, na intenção de continuar estudando em uma Instituição de Ensino Superior? Com isso, o objetivo desse estudo consiste em verificar se os fatores das variáveis espaciais, em interação com os aspectos pedagógicos e estruturais, influenciam no nível de satisfação dos alunos e, na intenção de continuar estudando em uma Instituição de Ensino Superior.

O estudo justifica-se pela necessidade de estudos relacionados à avaliação de satisfação de alunos em IES, para que com base nisso, as instituições desenvolvam suas estratégias para a gestão e políticas de ensino. Conforme Walter, Tontini e Domingues (2006) “a avaliação da qualidade dos produtos e serviços de uma IES sob o ponto de vista de seus clientes e o estudo do impacto do desempenho desses atributos na satisfação dos alunos, ainda carecem de pesquisas".

Este estudo é relevante para a sociedade em geral, pois o diagnóstico pode priorizar investimentos de melhorias em características que impactam de forma otimizada em nível de satisfação dos acadêmicos. É interessante para a IES em estudo, pois pode colaborar com sua 
competitividade, bem como com sua manutenção financeira para a continuidade na prestação de serviços educacionais.

\section{REFERENCIAL TEÓRICO}

No referencial teórico são abordados aspectos relacionados à gestão universitária, satisfação dos alunos, variáveis espaciais e alguns estudos anteriores relacionados à satisfação dos alunos, a fim de embasar e dar suporte esta pesquisa.

\subsection{GESTÃO UNIVERSITÁRIA}

No contexto do ensino superior brasileiro, a década de 90 foi um grande marco em que foram superadas as barreiras para novas oportunidades no ambiente educacional, a partir de então, o ensino passou a ser considerado um negócio e, com isso, começou a disputa de mercado (MAINARDES; MIRANDA; CORREIA, 2011). Os autores destacam ainda que, do ponto de vista dos alunos, essa concorrência entre as IES possibilita a escolha, considerando vários aspectos, como preço, qualidade, conveniência, entre outros e, além disso, a melhoria do serviço prestado. Porém, do ponto de vista das instituições, o mercado competitivo traz a necessidade de uma gestão profissionalizada, com estratégias voltadas para o mercado.

Assim como qualquer outra organização, as instituições de ensino superior privadas necessitam de estratégias para atuarem com vantagem em num ambiente competitivo. Inseridas nesse contexto de competitividade em que as mudanças são constantes, cada vez mais necessitam se adaptar e sobreviver aos desafios e ameaças deste ambiente. Para isso, veem-se obrigadas a desenvolver estratégias com o objetivo de se consolidar e obter êxito em suas atividades (MEYER; MUGNOL, 2004).

Neste mesmo contexto, Meyer, Pascucci e Mangolin (2012) salientam que as IES brasileiras, têm enfrentado constantes desafios oriundos das mudanças no ambiente, caracterizado pela acirrada competição, fazendo com que necessitem rever sua gestão e com isso buscar formas mais eficientes e eficazes de atuação. Entre os diversos motivos, estão as constantes mudanças na política educacional, flutuações na demanda por cursos e as renovadas necessidades e expectativas dos alunos.

Como já visto, muitas são as dificuldades enfrentadas pelas instituições de ensino superior privadas, Machado (2008) destaca que dentre elas estão as dificuldades financeiras, mercadológicas, fidelização dos alunos, falta de estratégias bem definidas, dentre outros, essas 
dificuldades são agravadas com a falta de um modelo de gestão bem definido e de gestores competentes. Além disso, o autor enfatiza que como essas instituições estão inseridas em um mercado cada vez mais competitivo as dificuldades aumentam, com isso, somente as capacitadas conseguem sobreviver e crescer.

Uma importante estratégia para que uma instituição educacional seja de fato bemsucedida, é sua capacidade em lidar com seus públicos-alvo e gerar alto nível de satisfação a seus alunos, pois tendem a se tornar os melhores divulgadores da instituição. Assim, a satisfação mantém os alunos na instituição e, torna-se o meio mais eficaz para divulgar a qualidade e consolidar a imagem institucional, e consequentemente atrair maior número de acadêmicos (MEYER; MUGNOL, 2004).

\subsection{SATISFAÇÃO DOS ALUNOS}

No ambiente do mercado educacional, a qualidade dos serviços prestados e a satisfação dos alunos são fundamentais para a sobrevivência das IES (ALVES; RAPOSO, 2004). Em consonância a isso, Walter, Tontini e Domingues (2005, p. 58) consideram que a "satisfação do cliente de serviços está relacionada com o desempenho de vários fatores em várias dimensões. Deste modo, a satisfação dos alunos é fator crítico para o planejamento de ações de melhoria e manutenção da instituição". Por esse motivo, é relevante às instituições conhecer a satisfação dos seus alunos e, utilizar-se dessa informação para a gestão da universidade.

A importância da satisfação dos alunos, reside no fato de que por meio dela é possível alcançar êxito no ensino, garantir a permanência dos estudantes na instituição, além do que, possibilita uma avaliação positiva que é transmitida pelos alunos a outras pessoas. Nesse sentido, é importante encontrar maneiras confiáveis para medir a satisfação dos alunos, para que as instituições conheçam a sua realidade e possam comparar suas ações com as de outras IES e, acima de tudo, possam analisar seu desempenho ao longo do tempo (ALVES; RAPOSO, 2004).

A satisfação é um fator fundamental que garante a motivação do aluno no processo de formação acadêmica, pois interfere no seu aprendizado e, por consequência, na competência profissional quando inserido no mercado de trabalho. Assim, compreender quais variáveis influenciam a satisfação dos acadêmicos é primordial para as IES que prezam pela qualidade dos serviços prestados (VIEIRA; MILACH; HUPPES, 2008). 


\section{INFLUÊNCIA E DESEMPENHO DAS VARIÁVEIS ESPACIAIS EM INTERAÇÃO COM OS ASPECTOS PEDAGÓGICOS E ESTRUTURAIS NA SATISFAÇÃO DOS ALUNOS \\ DOI: http://dx.doi.org/10.5007/1983-4535.2018v11n1p92}

Na concepção de Alcântara et al. (2012), “a satisfação é um elemento importante para que se obtenha lealdade", desta forma, é importante para a instituição de ensino conhecer a satisfação dos seus alunos, para assim, desenvolver suas estratégias de atuação e competitividade. A literatura aborda alguns atributos que podem ser utilizados para verificar tal satisfação dos alunos. O Quadro 1 contempla alguns desses principais fatores observados:

Quadro 1 Categorias para verificar a satisfação dos alunos

\begin{tabular}{|c|c|}
\hline Categoria & Autores \\
\hline $\begin{array}{c}\text { Atendimento/Suporte } \\
\text { às atividades universitárias }\end{array}$ & Walter, Tontini e Domingues (2006); Alcântara et al. (2012) \\
\hline $\begin{array}{c}\text { Infraestrutural } \\
\text { Tangível }\end{array}$ & $\begin{array}{c}\text { Owlia e Aspinwall (1996); Walter, Tontini e Domingues } \\
\text { (2006); Alcântara et al. (2012) }\end{array}$ \\
\hline $\begin{array}{c}\text { Capacitação dos } \\
\text { professores }\end{array}$ & $\begin{array}{l}\text { Owlia e Aspinwall (1996); Walter, Tontini e Domingues } \\
\text { (2006); Vieira, Milach e Huppes (2008); Alcântara et al. (2012) }\end{array}$ \\
\hline Métodos de Ensino & Walter, Tontini e Domingues (2006); Vieira, Milach e \\
Huppes (2008); Alcântara et al. (2012)
\end{tabular}

Fonte: Elaborado pelos autores

Em relação ao atendimento ou suporte às atividades universitárias, são definidos nesta categoria o atendimento da prestação de serviços ao acadêmico tais como a gentileza, cortesia e prestatividade no atendimento da secretaria, facilidade de acesso à coordenação do curso, agilidade no laboratório de impressão, atividades de monitoria, dentre outros (WALTER; TONTINI; DOMINGUES, 2006; ALCÂNTARA et al., 2012).

Os tangíveis ou a infraestrutura são representados pela quantidade e a qualidade de equipamentos e instalações, tais como laboratórios, biblioteca e equipamentos de informática, desempenham um papel fundamental na aprendizagem, bem como, nos processos de ensino. Além disso, os serviços de apoio como alojamentos, centros de esportes, restaurantes, e do ambiente em geral também devem ser incluídos nesta dimensão (OWLIA; ASPINWALL, 1996). Acrescentam-se ainda fatores como a quantidade de títulos da biblioteca, a modernidade dos laboratórios de informática e, a qualidade da estrutura física da sala de aula (WALTER, TONTINI; DOMINGUES, 2006; ALCÂNTARA et al., 2012).

No que se refere à capacitação ou competência dos professores, Owlia e Aspinwall (1996) argumentam ser um fator vital para o ensino superior e, está essencialmente 
relacionado com o conhecimento do pessoal acadêmico. Além disso, sua familiaridade com aplicações práticas, bem como, seus conhecimentos em técnicas de ensino devem ser incluídos. Walter, Tontini e Domingues (2006), Alcântara et al. (2012) e Vieira, Milach e Huppes (2008) complementam e acrescentam nessa categoria a atualização dos professores, a titulação ou qualificação destes.

Quanto aos métodos de ensino Walter, Tontini e Domingues (2006), Vieira, Milach e Huppes (2008) e Alcântara et al. (2012) consideram alguns fatores em comum que são importantes neste atributo, como a diversidade de métodos de ensino, a relação entre teoria e prática nas disciplinas, a quantidade de materiais no ambiente virtual de aprendizagem e os recursos didáticos utilizados pelos professores e, a experiência prática dos professores sobre o conteúdo ministrado.

O envolvimento ou atitudes do professor diz respeito a fatores como a percepção do aluno referente ao entusiasmo e interesse do professor, habilidades em explicar o conteúdo, utilizando-se de exemplos para que os alunos compreendam os assuntos (VIEIRA; MILACH; HUPPES, 2008). Além disso, Alcântara et al. (2012) consideram como componentes deste atributo fatores como o nível de exigência por parte dos professores, a capacidade de fazer-se compreender, comprometimento dos professores, a forma de ensino dos professores, tratamento dos professores aos alunos, dentre outros.

Owlia e Aspinwall (1996) acrescentam ao atributo de envolvimento ou atitudes do professor a compreensão das necessidades dos alunos, a vontade de ajudar, a disponibilidade para orientação, bem como, atenção dada ao aluno e simpatia. Nesta categoria cabem ainda os atributos de interação professor-estudante que é representado por fatores como a oportunidade do aluno discutir, questionar e esclarecer suas dúvidas durante as aulas, a liberdade para expressar os seus pontos de vista, bem como, o estímulo dos professores para isto. Outros fatores considerados neste atributo são a disponibilidade aos alunos dos materiais de estudo sobre as aulas dadas e as avaliações serem realizadas com precisão e justiça (VIEIRA; MILACH; HUPPES, 2008).

Para o conteúdo ou fatores extraclasse, Walter, Tontini e Domingues (2006) e Alcântara et al. (2012) atribuem como fatores que compõem esta categoria a contribuição do curso para o desempenho profissional, oferta de atividades extracurriculares e existência de empresa júnior, oportunidades de participar de projetos de ensino, pesquisa ou extensão, dentre outros. 
No atributo organização do curso Vieira, Milach e Huppes (2008) avaliam, entre outros aspectos, se os conceitos foram relacionados sistematicamente e a grade curricular do curso foi esboçada adequadamente. Owlia e Aspinwall (1996) levam em consideração a relevância do currículo para os futuros trabalhos dos estudantes, a eficácia ou efetividade, os conhecimentos e capacidades, o desenvolvimento de capacidades de comunicação e trabalho em equipe e, a interdisciplinaridade.

A credibilidade e, confiabilidade da instituição é normalmente baseada à atribuição de diplomas válidos, cumprimento das promessas e alcance das metas e, o tratamento das reclamações e solução dos problemas (OWLIA; ASPINWALL, 1996).

\subsection{VARIÁVEIS ESPACIAIS}

O cliente, ao ter uma experiência de compra em um determinado local como, por exemplo, um varejo, tem contato com variáveis atmosféricas, em que pode gerar reações de aproximação ou de afastamento (DONOVAN, 1982). Essas variáveis atmosféricas de influência estão representadas na Figura 1.

Figura 1 Influências Atmosféricas sobre a Experiência de Serviço

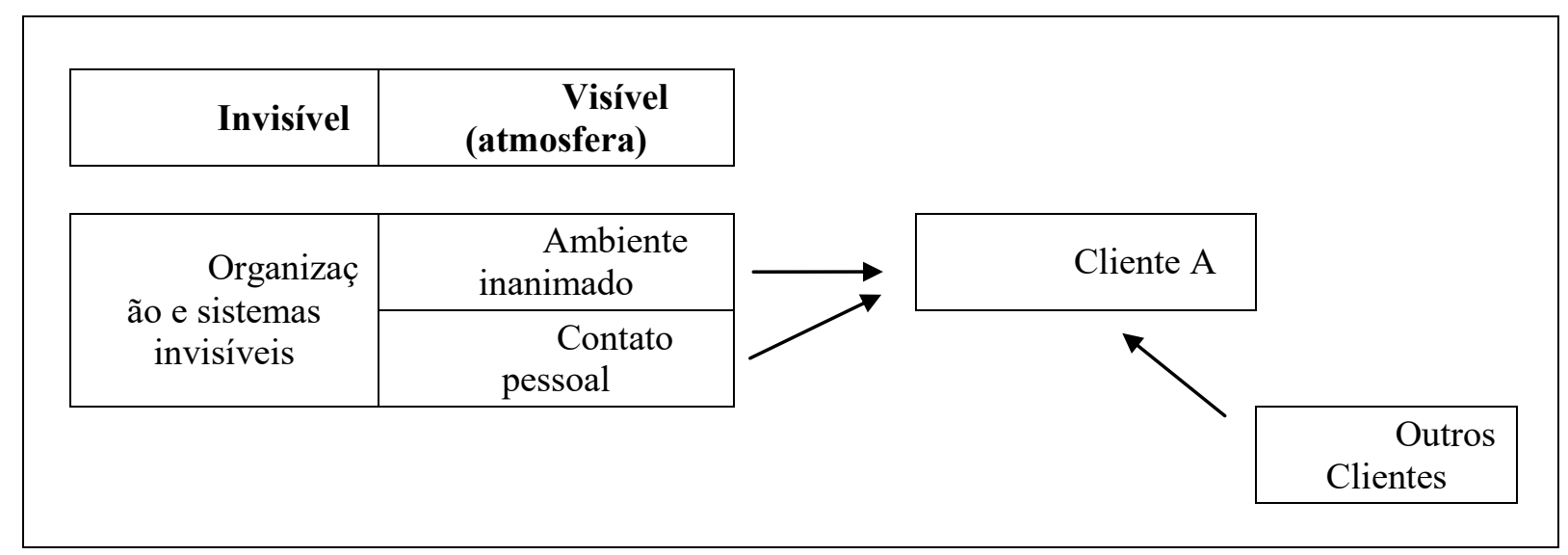

Fonte: Adaptado de Hoffman; Turley (2002).

As variáveis atmosféricas podem ser humanas, por meio do contato pessoal dos atendentes do estabelecimento comercial ou ainda, reações de outros clientes presentes no ambiente. Tem-se ainda, contato com o ambiente inanimado (HOFFMAN; TURLEY, 2002).

Pesquisadores de marketing têm observado que os consumidores são influenciados por estímulos físicos experimentados no momento de compra e a gestão do ambiente que possam 


\section{INFLUÊNCIA E DESEMPENHO DAS VARIÁVEIS ESPACIAIS EM INTERAÇÃO COM OS ASPECTOS PEDAGÓGICOS E ESTRUTURAIS NA SATISFAÇÃO DOS ALUNOS \\ DOI: http://dx.doi.org/10.5007/1983-4535.2018v11n1p92}

influenciar os clientes, deve ser uma estratégia importante, pois podem afastar ou aproximar clientes ou usuários de um serviço (TURLEY; MILLIMAN, 2000).

Turley e Milliman (2000) efetuaram uma revisão, comparando 60 estudos empíricos sobre efeitos atmosféricos relacionados com os consumidores, categorizando e identificando características comuns aos diversos estudos, conforme exposto no Quadro 2.

As variáveis externas incluem a loja, marquise entradas, arquitetura, estacionamento, e demais variáveis que possam influenciar os clientes na parte externa do estabelecimento. Estas variáveis merecem atenção, uma vez que é o primeiro conjunto a formar a impressão ao cliente, normalmente visto pelo consumidor, podendo atrair ou afastar instantaneamente, mas também no médio e longo prazo.

As variáveis internas, como revestimento de pisos ou carpetes, iluminação, perfumes e sons, podem influenciar nas vendas do estabelecimento comercial. As variáveis de layout e design incluem principalmente a alocação de espaços, dentro do departamento, fluxo de tráfego e locais de departamento.

Quadro 2 Variáveis espaciais

\begin{tabular}{|c|c|}
\hline 1. Variáveis Externas & $\begin{array}{l}\text { Sinais exteriores; Entradas; Vitrines Exteriores; Altura do edifício; } \\
\text { Tamanho da construção; Cor de prédio; Lojas circundantes; Os gramados e } \\
\text { jardins; Endereço e localização; Estilo arquitetônico; Zona envolvente; } \\
\text { Disponibilidade de estacionamento; Congestionamento e tráfego; As paredes } \\
\text { exteriores. }\end{array}$ \\
\hline 2. Variáveis Internas & $\begin{array}{l}\text { Pisos e carpetes; Os esquemas de cores; Iluminação; Música; Cheiro } \\
\text { característico; Fumo do tabaco; Largura de corredores; Composição da } \\
\text { parede; Pintura e papel de parede; Composição de teto; Merchandise; } \\
\text { Temperatura; Limpeza. }\end{array}$ \\
\hline $\begin{array}{l}\text { 3. Va } \\
\text { Layo }\end{array}$ & $\begin{array}{l}\text { Projeto do espaço e alocação; Colocação de mercadoria; } \\
\text { Agrupamento de mercadoria; Localização da estação de trabalho; Colocação } \\
\text { de equipamentos; Colocação de caixas registradoras; Áreas de espera; Salas } \\
\text { de espera; Localizações departamento versus fluxo de tráfego; Prateleiras e } \\
\text { caixas; Mobiliário; Áreas mortas. }\end{array}$ \\
\hline $\begin{array}{l}\text { Venda e } \\
\text { is de }\end{array}$ & $\begin{array}{l}\text { Displays de Ponto de venda; Sinais e cartões; Decorações de paredes; } \\
\text { Diplomas e certificados; Imagens; Obra de arte; Exposição de produtos; } \\
\text { Instruções de uso; Displays de preços; Teletexto. }\end{array}$ \\
\hline 5. Variáveis Humanas & $\begin{array}{l}\text { Características dos empregados; Uniformes dos empregados; } \\
\text { Aglomera mento de pessoas, superlotação; Características dos clientes; } \\
\text { Privacidade. }\end{array}$ \\
\hline
\end{tabular}

Fonte: Adaptado de Turley e Milliman (2000)

Os pontos de compra e decoração incluem a exposição dos produtos, displays, cartazes e mensagens, o quanto a exposição dos produtos podem influenciar as vendas e, a atratividade dos consumidores. Por fim, as variáveis humanas, inclui a aglomeração, densidade, 
privacidade, características dos funcionários bem como dos outros consumidores (TURLEY; MILLIMAN, 2000; PARENTE, 2000; MORETTO, 2007).

\section{PROCEDIMENTOS METODOLÓGICOS}

O estudo caracteriza-se, quanto aos objetivos, como descritiva, pois descreve se os fatores das variáveis espaciais, em interação com os aspectos pedagógicos e estruturais, influenciam no nível de satisfação dos alunos e, na intenção de continuar estudando em uma Instituição de Ensino Superior. Em relação aos procedimentos, remete-se a um levantamento pelo fato de levantar os dados em uma IES. No que se refere a abordagem do problema, tratase de uma pesquisa quantitativa, devido ao uso de instrumentos estatísticos nas fases de coleta, tratamento e análise dos dados.

\subsection{POPULAÇÃO E AMOSTRA}

A população compreende os acadêmicos de graduação que estão finalizando o curso de administração com habilitação em Comércio Exterior de uma Instituição de Ensino Superior de Santa Catarina. A população da pesquisa é intencional e não probabilística escolhida devido a facilidade de acesso aos dados. A amostra da pesquisa é representada por 61 alunos que concordaram em participar da pesquisa, tomando tempo suficiente para responder as questões formuladas.

A escolha desta população justifica-se por representar os períodos concluintes do curso, pois estão a mais tempo na instituição de ensino e conhecem melhor suas particularidades e características, desta forma, acredita-se que tenham melhores condições de explicitar sua opinião sobre os aspectos a serem analisados.

\subsection{COLETA E ANÁLISE DOS DADOS}

A coleta de dados se deu em maio de 2014. Por meio do questionário, foi identificada a importância de dez dimensões de possível influência na satisfação do acadêmico, enquanto usuário de um serviço de ensino, conforme descrito no Quadro 3. 
Quadro 3 Dimensões avaliadas

\begin{tabular}{|l|l|l|}
\hline CÓD & GRUPO & Significado \\
\hline D1 & $\begin{array}{l}\text { Atendimento, suporte às } \\
\text { atividades universitárias. }\end{array}$ & $\begin{array}{l}\text { Gentileza e cortesia no atendimento da secretaria, facilidade de } \\
\text { acesso à coordenação do curso, agilidade da central de impressões. }\end{array}$ \\
\hline D2 & Qualidade dos professores & $\begin{array}{l}\text { Capacitação, métodos de ensino, atitude, envolvimento do professor } \\
\text { e interação professor-estudante. }\end{array}$ \\
\hline D3 & $\begin{array}{l}\text { Organização das } \\
\text { disciplinas do curso }\end{array}$ & $\begin{array}{l}\text { Grade curricular adequada, atividades complementares (cursos, } \\
\text { palestras), relevância das disciplinas do curso para o desempenho } \\
\text { profissional, atualidade das disciplinas. }\end{array}$ \\
\hline D4 & $\begin{array}{l}\text { Credibilidade da } \\
\text { Instituição de ensino }\end{array}$ & $\begin{array}{l}\text { Aceitação da instituição na sociedade em geral, cumprimento das } \\
\text { promessas. }\end{array}$ \\
\hline D5 & Infraestruturas Tangíveis & $\begin{array}{l}\text { Quantidade de vagas no estacionamento, quantidade de títulos na } \\
\text { biblioteca, qualidade dos laboratórios de informativa. }\end{array}$ \\
\hline D6 & $\begin{array}{l}\text { Variáveis espaciais } \\
\text { externas }\end{array}$ & $\begin{array}{l}\text { Fachada da instituição, estilo arquitetônico, área circundante da } \\
\text { instituição, pintura externa, gramados e jardins. }\end{array}$ \\
\hline D7 & $\begin{array}{l}\text { Variáveis espaciais } \\
\text { interiores }\end{array}$ & $\begin{array}{l}\text { Pisos, pintura interna, iluminação, odor/ aroma, ruídos, temperatura e } \\
\text { limpeza. }\end{array}$ \\
\hline D8 & $\begin{array}{l}\text { Variáveis espaciais em } \\
\text { relação desenho do layout }\end{array}$ & $\begin{array}{l}\text { Localização das salas, alocação de espaços, localização dos centros } \\
\text { de coordenação, bibliotecas e cantinas. }\end{array}$ \\
\hline D9 & $\begin{array}{l}\text { Variáveis espaciais de } \\
\text { comunicação visual }\end{array}$ & $\begin{array}{l}\text { Comunicação visual de localização dos setores, blocos, salas de aula, } \\
\text { murais. }\end{array}$ \\
\hline D10 & $\begin{array}{l}\text { Variáveis espaciais } \\
\text { humanas }\end{array}$ & $\begin{array}{l}\text { Aglomeração de pessoas, aparência pessoal dos colegas, professores } \\
\text { e funcionários, tipos de roupas / uniformes. }\end{array}$ \\
\hline
\end{tabular}

Fonte: Dados da pesquisa

As cinco primeiras dimensões referem-se a atendimento e suporte às atividades universitárias, qualidade dos professores, a organização das disciplinas do curso, a credibilidade da instituição de ensino e a infraestrutura tangível da instituição. As demais dimensões referem-se às variáveis físico-espaciais sendo elas as variáveis externas, variáveis internas, design e layout, comunicação visual e humana.

A primeira parte do questionário são questões fechadas e refere-se ao perfil dos respondentes quanto ao gênero, faixa etária e renda familiar. A segunda parte, refere-se à mensuração do nível médio de satisfação dos acadêmicos em relação a dez dimensões e, o nível de satisfação com o desempenho geral da instituição. Os níveis de satisfação foram obtidos com escala de likert que variam de 1 a 8 , com rótulos de escala que variam de "extremamente insatisfeito" a "extremamente satisfeito". Para a análise dos dados, as avaliações foram recodificadas para variar de -1 a +1 e os níveis médios de satisfação estão apresentados na Tabela 3.

Com os níveis de satisfação das dimensões, foi efetuada a análise PRC. A análise do PRC consiste em ajustar uma regressão múltipla com variáveis dummy entre o nível de 
satisfação com o desempenho geral da instituição (variável dependente) e o desempenho dos atributos ( $\mathrm{Y}=\square 0+\square$ XX1neg $+\square 2 \mathrm{X} 1$ pos $+\square$ nXnneg $+\square$ nXnpos). O pressuposto com esse método, é que o importante é o nível de satisfação geral com a instituição em estudo. Assim, dimensões que influenciam o nível de satisfação geral, são consideradas relevantes, observando a interação entre as diversas variáveis independentes (PICOLO, 2005; MATZLER et al, 2004; ANDERSON; MITTAL, 2000).

O desempenho de cada dimensão foi recodificado em duas variáveis dummy. Quando o desempenho da dimensão está acima de determinada referência, a variável dummy recompensa (Xnpos) é o gap entre a avaliação e o valor de referência e, a variável dummy penalidade (Xnneg) é igual a zero. Se o desempenho for abaixo da referência, a variável dummy penalidade é o gap entre a referência e a avaliação da dimensão e, a Xnpos igual a zero (Tabela 1). Quando o desempenho é igual a referência, ambas variáveis Xnpos e Xnneg são iguais a zero. Nesse estudo, o valor de referência foi igual ao nível de satisfação médio atual de cada dimensão pesquisada, para verificar a influência de cada dimensão na satisfação geral no acréscimo ou decréscimo do desempenho atual das dimensões (PICOLO; TONTINI, 2008).

Tabela 1 Exemplo de recodificação PRC com o desempenho médio como referência

\begin{tabular}{|c|c|c|c|c|c|}
\hline \multirow[b]{2}{*}{ Respondente } & \multicolumn{2}{|c|}{ Dados da pesquisa } & \multicolumn{3}{|c|}{$\begin{array}{l}\text { Dimensões recodificadas com variáveis } \\
\text { dummy }\end{array}$} \\
\hline & $\begin{array}{l}\text { Satisfação } \\
\text { com a } \\
\text { dimensão }\end{array}$ & $\begin{array}{l}\text { Satisfação com o } \\
\text { desempenho geral } \\
\text { da IES }\end{array}$ & Penalidades & Recompensas & $\begin{array}{l}\text { Satisfação com o } \\
\text { desempenho geral } \\
\text { da IES }\end{array}$ \\
\hline 01 & $000^{+2,0}$ & $+1,0000$ & $000^{0,0}$ & $667^{+1,6}$ & $+1,0000$ \\
\hline 02 & $1,0000^{-}$ & $-1,0000$ & $1,3333^{-}$ & 0,000 & $-1,0000$ \\
\hline 03 & $00^{0,00}$ & 0,0000 & $000^{0,0}$ & $3^{0,333}$ & 0,0000 \\
\hline ia & $333^{+0,3}$ & 0,0000 & $4_{4444^{+0}}$ & $666^{+0,6}$ & 0,0000 \\
\hline
\end{tabular}

Fonte: Adaptado de Picolo e Tontini (2008)

Foi utilizado o método stepwise com p-enter igual a 0,05 e p-remove igual a 0,10 . Obteve-se assim, dois coeficientes de regressão para uma única variável independente. $\mathrm{O}$ coeficiente "recompensas", representando o impacto na variável dependente se o desempenho da variável independente for melhorado e, o coeficiente "penalidades" representando o impacto na variável dependente se tiver o desempenho reduzido (Tabela 3). O coeficiente $\square 0$ 
é igual a 4,367. O R2 ajustado ficou em 0,618 mostrando resultado de ajuste maior que 0,50 apresentando nível satisfatório para ciências sociais (MAROCO, 2003). Quanto à normalidade dos resíduos, a significância do teste Kolmogorov - Smirnov foi de p-value $=$ 0,145, não se pode rejeitar a hipótese de ter uma distribuição normal. O Teste VIF teve valor mínimo de 1,086 e máximo de 1,353, demonstra não apresentar problemas de multicolinearidade.

A terceira parte do questionário avalia o nível de importância das dimensões segundo o modelo Kano (1984). Para cada dimensão analisada, duas perguntas foram efetuadas, uma pergunta verificando qual o nível de satisfação esperado, caso tenham o seu desempenho melhorado e, outra pergunta, verificando o nível de insatisfação caso o desempenho da dimensão seja reduzido. A relevância da dimensão é medida pela capacidade de gerar satisfação com sua suficiência ou insatisfação com sua insuficiência. Os níveis de satisfação com a suficiência podem ser maiores do que os níveis de satisfação com a ausência, sendo considerados atrativos, podem ser iguais, sendo classificados como unidimensionais, ter desempenhos menores, sendo classificados como obrigatórios, ou ainda, serem insignificantes neutros (TONTINI, PICOLO, 2010). Os níveis de satisfação e insatisfação esperados foram efetuados com escala de likert que variam de 1 - "extremamente insatisfeito" a 8 "extremamente satisfeito" e as avaliações foram recodificadas para variar de $-1 \mathrm{a}+1$ (Tab. 3).

A quarta parte, identifica a importância declarada dos respondentes, por meio do escalonamento constante, em relação às dimensões analisadas. Os entrevistados atribuíram uma soma constante de 100 pontos às diversas dimensões que refletissem a importância nos diversos atributos analisados (MALHOTRA, 2011). Quando o entrevistado declarou pontos onde a soma foi diferente de 100 pontos, os valores foram ajustados para ter soma de 100 pontos e somente depois foi calculada a média das dimensões e apresentadas na Tabela 3.

\section{DESCRIÇÃO E ANÁLISE DOS DADOS}

Nesta seção, é apresentada a análise dos dados da pesquisa. A Tabela 2 apresenta o perfil dos respondentes. 
Tabela 2 Perfil dos entrevistados

\begin{tabular}{c|c|c|c|c}
\hline Gênero & $\%$ & CS & Renda Mensal Familiar & \% \\
\hline Masculino & 34,43 & E & Até R $\$ 1.448,00$ & 19,03 \\
Feminino & 65,57 & D & De R\$ 1.449,00 a R $\$ 2.896,00$ & 29,51 \\
Faixa Etária & $\%$ & C & De R $\$ 2.897,00$ a R $7.240,00$ & 40,98 \\
20 a 25 anos & 75,41 & A $/$ B & Acima de $7.241,00$ & 19,54 \\
26 anos ou mais & 24,59 & - & Não sabe & 8,20 \\
\hline
\end{tabular}

Fonte: Dados da pesquisa

De acordo com a Tabela 2, o perfil dos respondentes, quanto ao gênero, é predominante feminino, corresponde a $65,57 \%$, do gênero masculino são $34,43 \%$. Quanto a faixa etária a maioria tem entre 20 a 25 anos, $75,41 \%$ e, com 26 ou mais anos são $24,59 \%$. No que se refere a classe social, verifica-se que a predominante é classe C que representa 40,98\%, com uma renda familiar mensal de $\mathrm{R} \$ 2.897,00$ a $\mathrm{R} \$ 7.240,00$.

A fim de analisar a importância das variáveis físico-espaciais nas instituições de ensino superior, em interação aos aspectos pedagógicos e estruturais, foram analisados os resultados contidos na Tabela 3 .

Tabela 3 Resultado da pesquisa

\begin{tabular}{l|l|c|c|c|c|c|c|c}
\hline \multirow{2}{*}{ CóD } & \multicolumn{1}{|c|}{ GRUPO } & \multirow{2}{*}{$\begin{array}{c}\text { Nível de } \\
\text { Satisfação }\end{array}$} & $\begin{array}{c}\boldsymbol{\beta} \\
\text { atual }\end{array}$ & $\begin{array}{c}\boldsymbol{\beta} \\
\text { rec }\end{array}$ & $\begin{array}{c}\text { Satisfação } \\
\text { pen }\end{array}$ & $\begin{array}{c}\text { Insatisfação } \\
\text { Esperada }\end{array}$ & $\begin{array}{c}\text { Soma } \\
\text { Esperstante }\end{array}$ \\
\hline D1 & $\begin{array}{l}\text { Atendimento, suporte às } \\
\text { atividades universitárias. }\end{array}$ & 0,157 & 0,000 & 0,000 & 0,377 & $-0,595$ & 11,364 \\
\hline D2 & Qualidade dos professores & 0,166 & 0,518 & 0,000 & 0,657 & $-0,746$ & 22,473 \\
\hline D3 & $\begin{array}{l}\text { Organização das disciplinas do } \\
\text { curso }\end{array}$ & $-0,002$ & 0,000 & 0,000 & 0,574 & $-0,688$ & 15,563 \\
\hline D4 & $\begin{array}{l}\text { Credibilidade da instituição de } \\
\text { ensino }\end{array}$ & 0,349 & 0,477 & 0,000 & 0,574 & $-0,693$ & 10,979 \\
\hline D5 & Infraestruturas tangíveis & $-0,026$ & 0,000 & 0,000 & 0,543 & $-0,642$ & 10,081 \\
\hline D6 & Variáveis espaciais externas & 0,218 & 0,584 & 0,000 & 0,497 & $-0,524$ & 5,539 \\
\hline D7 & Variáveis espaciais interiores & 0,016 & 0,000 & 0,000 & 0,439 & $-0,496$ & 6,274 \\
\hline D8 & Variáveis espaciais do layout & 0,133 & 0,000 & 0,000 & 0,439 & $-0,470$ & 6,577 \\
\hline D9 & $\begin{array}{l}\text { Variáveis espaciais de } \\
\text { comunicação visual }\end{array}$ & 0,129 & 0,000 & 0,000 & 0,381 & $-0,497$ & 5,400 \\
\hline D10 & Variáveis espaciais humanas & 0,222 & 0,000 & 0,362 & 0,506 & $-0,605$ & 5,749 \\
\hline- & Desempenho geral & 0,136 & - & - & - & - & - \\
\hline- & Média & 0,101 & 0,158 & 0,036 & 0,499 & $-0,596$ & 10,000 \\
\hline
\end{tabular}

Fonte: Dados da pesquisa

Quanto ao desempenho atual da instituição de ensino superior conforme apresentado na Tabela 3, a dimensão que apresenta o maior nível de satisfação é D4 "credibilidade da 
instituição de ensino"; seguida de D10 - "variáveis espaciais humanas"; D6 - "variáveis espaciais externas"; D2 - "qualidade dos professores"; D1 - "atendimento, suporte das atividades universitárias"; D8 - "variáveis espaciais de layout"; D9 - "variáveis espaciais de comunicação visual e D7 - "variáveis espaciais interiores". E as dimensões que apresentam os menores desempenhos são D3 - "organização das disciplinas do curso" e D5 infraestruturas tangíveis".

A análise PRC, revelou dimensões consideradas importantes por influenciar a satisfação geral, mas demonstrou uma relação não linear. As dimensões com coeficientes de regressão de recompensa são a D2 - "qualidade dos professores"; D4 - "credibilidade da instituição de ensino" e D6 - "variáveis físico-espaciais externas". Essas dimensões influenciam positivamente a satisfação geral, apresentando recomendação gerencial de manter o desempenho das respectivas dimensões sempre elevadas, se possível incrementar ainda mais seus desempenhos atuais, porque influenciam positivamente o nível de satisfação geral com o curso. A dimensão com coeficiente penalidade é a dimensão D10 - "variáveis físico-espaciais humanas". A recomendação gerencial é manter o desempenho atual, pois se o nível de satisfação for reduzido, influencia negativamente a satisfação geral com o curso em estudo.

Quanto aos níveis de satisfação obtidos pelas questões, segundo o modelo Kano et al. (1984), tanto os níveis de satisfação e insatisfação esperada em função da suficiência e insuficiência das dimensões, as dimensões consideradas mais importantes, por estarem acima do desempenho médio das diversas dimensões são D2 - "qualidade dos professores"; D3 "organização das disciplinas do curso"; D4 - "credibilidade da instituição de ensino"; D5 “infraestruturas tangíveis e uma única variável espacial, a D10 - variável espacial humana.

Por fim, a importância declarada pelos respondentes, com importâncias superiores são as dimensões D1 - "atendimento, suporte às atividades universitárias", D2 - qualidade dos professores; D3 - organização das disciplinas do curso; D4 - credibilidade da instituição de ensino; D5 - "infraestruturas tangíveis" e as demais dimensões, incluindo todas as dimensões relacionadas as variáveis espaciais, não foram declaradas importantes pelos respondentes.

\subsection{COMPARATIVOS DAS IMPORTÂNCIAS DECLARADAS}

A fim de comparar a importância dos diversos métodos, sendo eles, o método PRC, Modelo Kano e Soma constante, tiveram seus valores padronizados e apresentados na Figura 2. Quanto a análise PRC, os coeficientes positivos recodificados representam as dimensões 
que influenciam a satisfação geral, independente do coeficiente penalidade ou recompensa (Equação 1). Os coeficientes recodificados negativos estão alocados nas dimensões que não influenciam a satisfação geral com o curso. Os níveis de satisfação esperada (Equação 2), recodificados positivos, representam as dimensões que mais geram satisfação, sendo valores acima de 0,499 que é a média de todas as dimensões. O nível de insatisfação esperada (Equação 3), os valores positivos representam as dimensões que mais geram insatisfação (abaixo de -0,596), caso tenha seu desempenho reduzido e, a importância declarada recodificada positiva, representa as dimensões que tiveram importância declarada acima da média de $10,00 \%$.

Coeficiente $_{\mathrm{n}}=\frac{\left(\beta p e n_{\mathrm{n}}+\beta r e c n\right)-\overline{\mathrm{SE}}}{\sigma} \quad($ Equação 1$)$

$\mathrm{SE}_{\mathrm{n}}=\frac{\mathrm{SE}_{\mathrm{n}}-\overline{\mathrm{SE}}}{\sigma} \quad($ Equação 2$)$

$\mathrm{I} E_{\mathrm{n}}=\frac{\overline{\mathrm{IE}}-\mathrm{IE}_{\mathrm{n}}}{\sigma} \quad$ (Equação 3$)$

$\mathrm{S} C_{\mathrm{n}}=\frac{\mathrm{S} C_{\mathrm{n}}-\overline{\mathrm{SC}}}{\sigma} \quad($ Equação 4$)$

Comparando as importâncias, observa-se divergência nas diferentes dimensões. Entende-se que são diferentes porque os pressupostos de importância são diferentes. A importância determinada pela magnitude dos coeficientes de regressão pela análise PRC define se o desempenho de cada dimensão que influencia ou não na satisfação geral do curso, observando a interação das diversas dimensões (PICOLO; TONTINI, 2008). Entretanto, sendo uma análise de regressão, a existência de uma relação significativa estatisticamente, entre variável independente e dependente, não significa uma relação de causa e efeito (TONTINI; WALTER, 2011). 
Figura 2 Comparativos das importâncias das dimensões

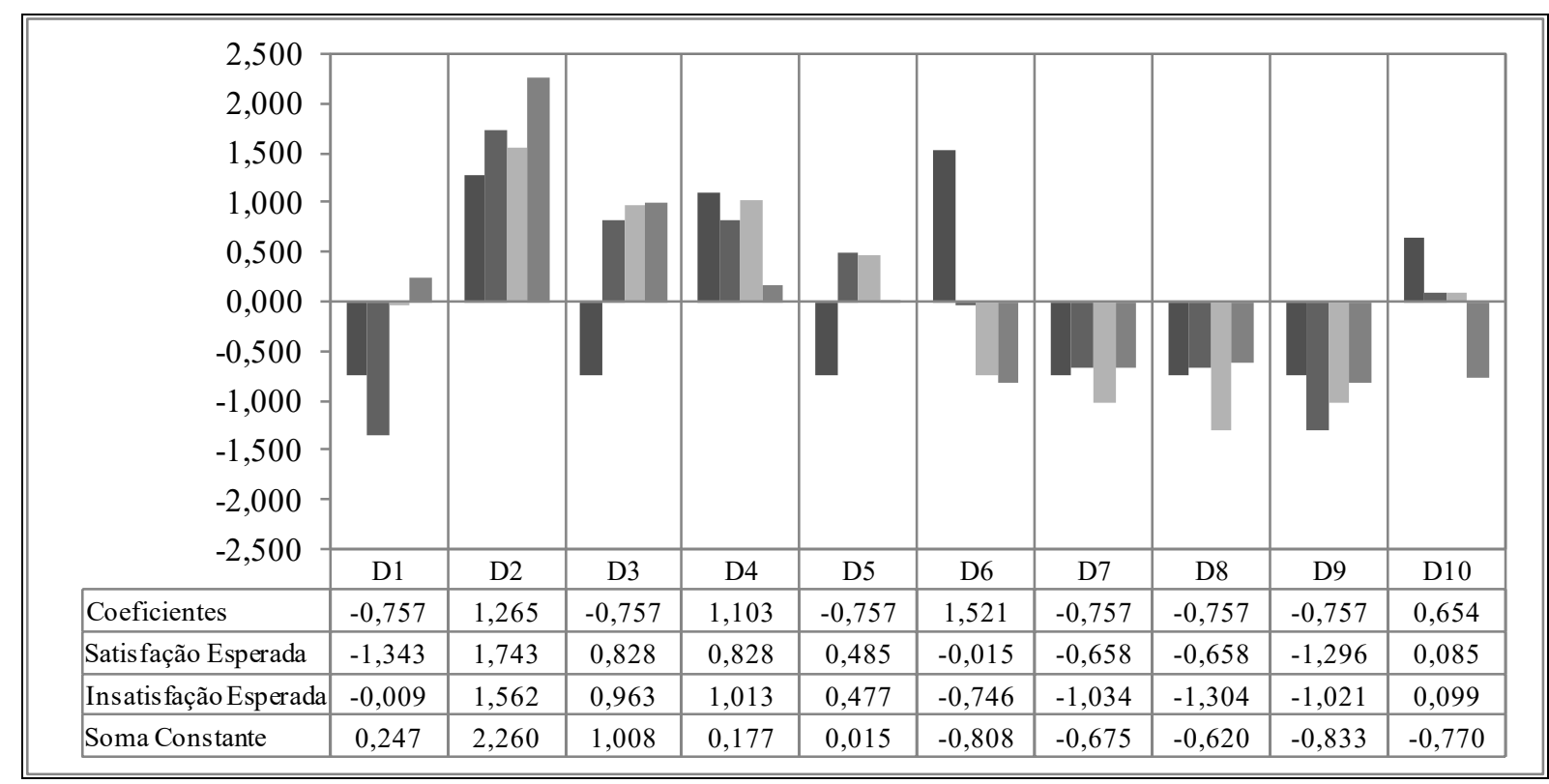

Fonte: Dados de pesquisa

A importância medida pelo nível de satisfação e insatisfação esperada é definida pela capacidade de cada dimensão gerar satisfação ou insatisfação, se cada dimensão tivesse seu máximo ou mínimo desempenho respectivamente. O intuito é verificar a capacidade da dimensão em gerar satisfação, para verificar se é interessante direcionar esforços de melhorias naquela dimensão ou, se é possível reduzir o desempenho, sem gerar insatisfação. Entretanto, apresenta como limitação, que não se pode saber se o nível de satisfação gerada com essa dimensão influencia ou não satisfação geral. Pode ocorrer que o respondente esteja satisfeito com determinada dimensão, mas não tenha impacto com a satisfação geral (PICOLO, 2005).

A importância declarada pode ter limitações. Uma das limitações pode ser interpretação do cliente quanto ao significado de importância, ao medir a importância, por exemplo, de um refrigerador, o atributo capacidade de refrigeração pode ser considerado importante, mas possivelmente não serão determinantes na diferenciação do produto, podendo ser observado outros aspectos como tamanho, design, marca ou cor, apesar de que não se pode descartar a importância como atributo obrigatório de um refrigerador (FUCHS; WEIERMAIR, 2004; MATZLER; SAURWEIN, 2002; PICOLO, 2005). Entretanto, na análise deve-se ter em mente, que atributo declarado como importante não necessariamente é um diferencial. 
A dimensão 1 - "atendimento, suporte às atividades universitárias" não influencia a satisfação geral, e não apresenta satisfação e insatisfação esperada significativa, mas foi declarado importante pelos respondentes, podendo ser um exemplo de limitação da importância declarada. Essa dimensão correspondente a gentileza e cortesia no atendimento da secretaria, facilidade de acesso a coordenação do curso e agilidade da central de impressões, por exemplo, podem ser considerados como questões elementares pelos acadêmicos e, devem ser atendidas, mas não necessariamente é um diferencial para os acadêmicos. Visto que o desempenho atual dessa dimensão é satisfatório, recomenda-se manter o padrão atual de desempenho, mas esforços de melhoria nessa dimensão, possivelmente não trazem diferenciais ao curso da IES analisado.

A dimensão 2 - "qualidade dos professores" e a dimensão 4 - "credibilidade da instituição de ensino" apresentaram alta importância por todos os métodos de importância utilizados. Essa congruência de importâncias gera maior certeza na melhoria dessas dimensões. Tanto gera satisfação esperada se o desempenho receber esforços de melhorias, com alta insatisfação de o desempenho for reduzido, também é declarado importante pelos respondentes. Apesar de apresentar desempenho satisfatório, recomenda-se incrementar esforços de melhorias nessa dimensão, uma vez que é um diferencial significativo ao curso.

A dimensão 3 - "organização das disciplinas do curso" e a dimensão 5 "infraestruturas tangíveis" não tiveram relação significativa com a satisfação geral do curso. Mas ambas as dimensões apresentaram desempenhos insatisfatórios, devendo ter seus desempenhos melhorados, pois apresentam capacidade de gerar satisfação ou insatisfação se tiver seu desempenho incrementado ou reduzido e foi declarado importante.

Em relação a importância das diversas variáveis física-espaciais, que é o objetivo principal de estudo deste artigo, as dimensões 7 - "variáveis interiores", dimensão 8 - "design e layout, e dimensão 9 - "comunicação visual não foram considerados importantes por nenhum método de importância. Como o desempenho atual é satisfatório, recomenda-se manter, mas entendendo que não é prioridade atual.

Quanto a dimensão 6 - "variáveis externas", apresentou relação entre o seu desempenho e a satisfação geral, apesar de que não apresenta satisfação ou insatisfação esperada significativas e, não foi declarado importante, pode ser considerado uma importância implícita, onde os respondentes não têm consciência de sua importância, mas pode influenciar a satisfação geral caso tenha seu desempenho melhorado. Recomenda-se, apesar do 
desempenho satisfatório com esse atributo, dar atenção, por exemplo, a fachada da instituição, ao estilo arquitetônico, área circundante da instituição, pintura externa, gramados e jardins.

A dimensão 10 - "variável espacial humana", influencia a satisfação geral e apresenta capacidade de gerar satisfação com a dimensão, apesar de não ser declarado pelos respondentes. Apesar do bom desempenho atual dessa dimensão, recomenda-se observar questões relacionadas às variáveis espaciais humanas, como por exemplo, o aglomerado de pessoas, aparência pessoal dos colegas, professores e funcionários, tipos de roupas e uniformes.

\section{CONSIDERAÇÕES FINAIS}

Este estudo teve como objetivo verificar se os fatores das variáveis espaciais, em interação com os aspectos pedagógicos e estruturais, influenciam no nível de satisfação dos alunos e, na intenção de continuar estudando em uma Instituição de Ensino Superior. Para isso, analisou-se as respostas de 61 alunos do curso de administração com habilitação em Comércio Exterior, de uma Instituição de Ensino Superior de Santa Catarina, avaliando 10 dimensões observadas a partir da literatura.

A partir de toda a análise dos dados, os principais achados foram que a dimensão "Atendimento, suporte às atividades universitárias" (D1) que representa a gentileza e cortesia no atendimento da secretaria, facilidade de acesso à coordenação do curso, agilidade da central de impressões, não apresentou influência na satisfação geral, tampouco apresenta satisfação e insatisfação esperada significativa, porém, foi declarada importante pelos respondentes (WALTER; TONTINI; DOMINGUES, 2006; ALCÂNTARA et al., 2012).

A dimensão "Qualidade dos professores" (D2), que envolve a capacitação, métodos de ensino, atitude, envolvimento do professor e interação professor-estudante, apresentou alta importância em todos os métodos de importância utilizados para análise, e também foram declarados importante pelos respondentes. Outra dimensão que assim se apresentou, foi a "Credibilidade da instituição de ensino" (D4), que representa a aceitação da instituição na sociedade em geral e, o cumprimento das promessas (OWLIA; ASPINWALL, 1996; WALTER; TONTINI; DOMINGUES, 2006; ALCÂNTARA et al., 2012; VIEIRA; MILACH; HUPPES, 2008).

Por sua vez, as dimensões "Organização das disciplinas do curso" (D3) que compreende a grade curricular adequada, atividades complementares (cursos, palestras), 
relevância das disciplinas do curso para o desempenho profissional, atualidade das disciplinas (OWLIA; ASPINWALL, 1996; WALTER; TONTINI; DOMINGUES, 2006; ALCÂNTARA et al., 2012; VIEIRA; MILACH; HUPPES, 2008) e, a dimensão "Infraestruturas tangíveis" (D5) que envolve a quantidade de vagas no estacionamento, quantidade de títulos na biblioteca, qualidade dos laboratórios de informativa, não tiveram relação significativa com a satisfação geral do curso, mas foram declaradas importantes pelos alunos (OWLIA; ASPINWALL, 1996; WALTER; TONTINI; DOMINGUES, 2006; ALCÂNTARA et al., 2012).

No que se refere a importância das diversas variáveis física-espaciais, verificou-se que a dimensão "Variáveis interiores" (D7) que contempla aspectos dos pisos, pintura interna, iluminação, odor/ aroma, ruídos, temperatura e limpeza; a dimensão "Design e layout" (D8) que englobam a localização das salas, alocação de espaços, localização dos centros de coordenação, bibliotecas e cantinas; e a dimensão "Comunicação visual” (D9) composta pela comunicação visual de localização dos setores, blocos, salas de aula, murais; não foram considerados relevantes por nenhum método de importância.

Quanto a dimensão "Variáveis externas" (D6), que abrange aspectos da fachada da instituição, estilo arquitetônico, área circundante da instituição, pintura externa, gramados e jardins, verificou-se que apresentou relação entre o seu desempenho e a satisfação geral, porém, não foi declarado importante pelos respondentes. Por fim, observou-se que a dimensão "Variável espacial humana" (D10), que leva em consideração a aglomeração de pessoas, aparência pessoal dos colegas, professores e funcionários, tipos de roupas/uniformes, influencia a satisfação geral e apresenta capacidade de gerar satisfação com a dimensão, no entanto, não foi declarada como importante pelos respondentes.

Desta forma, conclui-se que os aspectos pedagógicos são os mais elementares e que mais influenciam na satisfação dos alunos desta instituição estudada. Os aspectos estruturais e espaciais, de maneira geral, têm relação com a satisfação, no entanto, não foram consideradas como importantes pelos alunos. Tais considerações são pertinentes para as IES ao planejar as suas ações e, na gestão como um todo.

As limitações desta pesquisa foram em relação à avaliação de apenas um curso na Instituição, desta forma, os resultados e conclusões estão relacionados com a percepção deste grupo. Avaliando outros cursos, outros resultados podem ser obtidos. Portanto, recomendamse futuros estudos em fatores mais detalhados de cada dimensão para um diagnóstico mais 
preciso, também, comparando diferentes instituições de ensino, ou até mesmo entre cursos distintos para verificar se as opiniões se equiparam. Espera-se que este estudo possa contribuir para os estudos da influência das variáveis físico atmosféricas em instituições de ensino, e assim, ampliar a importância desta percepção nas instituições de ensino.

\section{REFERÊNCIAS}

ALCÂNTARA, V. de C.; LUIZ, G. V.; FERREIRA, A. C.; TEODORO, S. A. S. Dimensões e determinantes da satisfação de alunos em uma instituição de ensino superior. REMark Revista Brasileira de Marketing, São Paulo, v. 11, n. 3, p. 193-220, set./dez. 2012.

ALVES, H.; RAPOSO, M. La medición de la satisfacción en la enseóanza universitaria: El ejemplo de la universidade da beirainterior. International Review on Public and Nonprofit Marketing, v. 1, n. 1, p. 73-88, 2004.

ANDERSON, E.W.; MITTAL, V. Strengthening the satisfaction-profit chain. Journal of Service Research, v.3, n. 2, p.107-120, nov. 2000.

ATZLER, K.; BAILOM, F.; HINTERHUBER, H. H.; RENZL, B; PICHLER, J.. The asymmetric relationship between attribute-level performance and overall customer satisfaction: a reconsideration of the importance-performance analysis. Industrial Marketing Management, v. 33, n. 4, p.271-277, 2004.

BALDRIDGE, V. J. et al. Alternatives Model of Governance in Higher Education. In: BIRNBAUM, Robert (org.) Organization and Governance in Higher Education. Massachussets: Ginn Custon Publishing, 1971.

BATESON, J. E.G; HOFFMAN, K. D. Marketing de serviços. 4. ed. Porto Alegre: Bookman, 2001.

COLOSSI, N.; CONSENTINO, A.; QUEIROZ, E.G. Mudanças no contexto do ensino superior no Brasil: uma tendência ao ensino colaborativo. DA FAE, v. 4, n. 1, p. 51-8, 2007.

DONOVAN, R J.; ROSSITER, J. R. Store atmosphere: an environmental psychology approach. Journal of retailing, v. 58, n. 1, p. 34-57, 1982.

FUCHS, M.; WEIERMAIR, K. Destination benchmarking: na indicator system's potential for exploring guest satisfaction. Journal of travel research. v. 42. p. 212-225, 2002.

HOFFMAN, K. D.; TURLEY, L.W. Atmospherics, service encounters and consumer decision making: an integrative perspective. Journal of marketing. 2002.

KANO, N.; SERAKU, N.; TAKAHASHI, F.; TSUJI, S. Attractive quality and must-be quality. The Journal of the Japanese Society for Quality Control, abr, p. 39-48, 1984.

MACHADO, L. E. Gestão Estratégica para instituições de ensino superior privadas. FGV Editora, 2008. 
MAINARDES, E. W.; MIRANDA, C. S.; CORREIA, C. H. A gestão estratégica de instituições de ensino superior: um estudo multicaso. Contextus-Revista Contemporânea de Economia e Gestão, v. 9, n. 1, p. p. 19-32, 2011.

MALHOTRA Naresh K. Pesquisa de marketing: uma orientação aplicada. 3. ed. Porto Alegre: Bookman, 2011.

MAROCO, João. Análise estatística: com utilização do SPSS. Machester: Edições Silabo, 2003.

MATZLER, K; SAUERWEIN, Elmar. The factor structure of customer satisfaction. International Journal of Service Industry Management. v.13. n.4, p. 314-332, 2002.

MEYER, V. J.; MUGNOL, G. Competição e estratégia no contexto das instituições de ensino superior privadas. Revista Diálogo Educacional, v. 4, n. 11, p. 1-13, 2004.

MEYER, V. J.; PASCUCCI, L.; MANGOLIN, L. Gestão estratégica: um exame de práticas em universidades privadas. RAP-Revista de Administração Pública, p. 49-70, 2012.

MIRANDA, C. M. S; DOMINGUES, M. J. C. de S. Razões para escolha de uma IES: uma abordagem sobre o perfil sócio-econômico de alunos interessados em cursar administração. ENANGRAD, v. 17, p. 67-70, 2006.

MORETTO, M. M. Aspectos fisico-espaciais, satisfação e preferência no comércio de varejo. 2007. 163 f. Dissertação (Mestrado em Programa de Pós-Graduação em Planejamento Urbano e Regional) - Faculdade de Arquitetura, Universidade Federal do Rio Grande do Sul, Porto Alegre, 2007.

MOURA, F. V., BATISTA, I. V. C. Instituições de Ensino Superior em Contabilidade e suas contribuições para a formação de Profissionais Socialmente Responsáveis. In: IV Congresso USP de Iniciação Científica em Contabilidade, São Paulo, SP, 2007. Anais...São Paulo FEA-USP, 2007,

OWLIA, M. S.; ASPINWALL, E. M. A framework for the dimensions of quality in higher education. Quality Assurance in Education, v. 4, n. 2, p. 12-20, 1996.

PARENTE, J. Varejo no Brasil. São Paulo: Atlas, 2000.

PICOLO, J. D. Influência do desempenho de atributos de produtos ou serviços na satisfação dos clientes: uma análise comparativa entre diferentes técnicas de pesquisa. 2005. 197 f. Dissertação (Mestrado em Administração) - Centro de Ciências Sociais Aplicadas, Universidade Regional de Blumenau, Blumenau, 2005.

PICOLO, J. D.; TONTINI; G. Análise do contraste da penalidade e da recompensa (PRC): identificando oportunidades de melhoria em um serviço. RAM, Rev. Adm. Mackenzie. 2008, vol.9, n.5, p. 35-58, 2008.

RICHARDSON, R. J. Pesquisa social: métodos e técnicas. 2. ed. São Paulo: Atlas, 1989.

SANDER, B. Consenso e Conflito: perspectivas analíticas na pedagogia e na administração da Educação. São Paulo, Pioneira, 1984. 
SOUKI, G. Q.; PEREIRA, C. A. Satisfação, motivação e comprometimento de estudantes de administração: um estudo com base nos atributos de uma instituição de ensino superior. In: XXVIII EnANPAD - Encontro Nacional Associação Nacional dos Programas de Pós-Graduação em Administração, 2004, Curitiba. Anais... Curitiba, v. 28, 2004.

TURLEY, L. W; MILLIMAN, E. Ronald. Atmospheric effects on shopping behavior: a review of the experimental evidence. Journal of Business Research, v. 49, n. 2, p. 193-211, 2000 .

VIEIRA, K. M.; MILACH, F. T.; HUPPES, D. Equações estruturais aplicadas à satisfação dos alunos: um estudo no curso de ciências contábeis da Universidade Federal de Santa Maria. Revista Contabilidade \& Finanças, v. 19, n. 48, p. 65-76, 2008.

WAlter, S. A.; TONTINI, G.; DOMINGUES, M. J. C. de S. Análise da Satisfação do Aluno para Melhoria de um Curso de Administração. Revista de Administração FACES Journal, v. 5, n. 2, 2006.

WALTER, S. A.; TONTINI, G.; DOMINGUES, M. J. C. S. Identificando oportunidades de melhoria em um curso superior através da análise da satisfação dos alunos. In: XXIX EnANPAD - Encontro Nacional Associação Nacional dos Programas de Pós-Graduação em Administração, 2005, Brasília. Anais... Brasília, v. 29, 2005.

YACUZZI, E.; MARTIN, F. Aplicación del método de kano en el diseño de un producto farmacêutico. Buenos Aires: Universidade del CEMA, 2002. Disponível em: $<$ http://www.cema.edu.ar/publicaciones/download/documentos/224.pdf $>$. Acesso em 14 set. 2004 . 